\title{
Estudos das Experiências do Usuário e da Qualidade de uso de Sistemas no LUQS
}

\author{
Elizabeth S. Furtado \\ Universidade de Fortaleza, \\ Fortaleza, CE, Brasil \\ elizabet@unifor.br
}

\author{
Marília S. Mendes \\ Universidade Federal \\ do Ceará, \\ Russas, CE, Brasil \\ marilia.mendes@ufc.br
}

\author{
Julio Guido O. \\ Militão \\ Universidade de \\ Fortaleza, \\ Fortaleza, CE, Brasil \\ militao@unifor.br
}

\author{
Daniel A. Chagas \\ Universidade de Fortaleza, \\ Fortaleza, CE, Brasil \\ daniel.helptech@gmail.com
}

\begin{abstract}
RESUMO
Neste artigo, são descritas as principais pesquisas realizadas em um laboratório de pesquisa de experiência do usuário (UX) e Interação Humano-Computador (IHC) desde o seu surgimento em 1999 em Fortaleza, CE. As pesquisas realizadas no Laboratório de estudos do Usuário e da Qualidade do uso de Sistemas (LUQS) e laboratórios parceiros produzem muitos resultados acadêmicos, que têm os seguintes beneficiários: os alunos, os programas universitários envolvidos e, especialmente, a Região Nordeste do Brasil. Destaca-se, também, o trabalho do grupo de pesquisa com empresas locais, realizando ações de evangelização UX / IHC nos processos das empresas, além de ações para aprimorar seus produtos.
\end{abstract}

\section{PALAVRAS-CHAVE}

Formação de estudantes e profissionais; Avaliação de UX / Usabilidade; Interação de áudio; Modelagem de Interface do Usuário; Qualidade de uso

\section{Introdução}

O Laboratório de pesquisa LUQS pertence ao Programa de Pesquisa e Pós-graduação em Informática Aplicada (PPGIA) da Universidade de Fortaleza, e foi criado em 1999.

As pesquisas realizadas no LUQS se situam na área de IHC, e concentram-se na investigação de diversos aspectos para a qualidade do processo de projeto da interação de sistemas interativos e a qualidade das experiências dos usuários nos sistemas em desenvolvimentos e/ou em avaliação e têm embasamentos científicos relevantes nos exercícios profissional e acadêmico dos alunos de Ciência da Computação e de cursos afins, bem como têm aplicações práticas para a inovação de processos de software e de produtos das empresas envolvidas. A coordenadora do laboratório, profa. Elizabeth Sucupira Furtado, juntamente com os pesquisadores colaboradores, realizam atividades de ensino e

Permission to reproduce or distribute, in whole or in part, material extracted from this work, verbatim, adapted or remixed, as well as the creation or production from the content of such work, is granted without fee for non-commercial use, provided that the original work is properly credited.

IHC 2019 - TRILHA FÓRUM DOS GRUPOS DE PESQUISA, Outubro 21-25, 2019, Vitória, Brasil. In Anais Estendidos do XVIII Simpósio Brasileiro sobre Fatores Humanos em Sistemas Computacionais. Porto Alegre: SBC.

(C) 2019 by the author(s), in accordance with the terms of the Creative Commons Attribution-NonCommercial 4.0 International Public License (CC BY-NC 4.0). pesquisa de caráter multidisciplinar de IHC com as áreas de Engenharia de Software, Inteligência Artificial (IA) e Pesquisa Operacional.

Foram 80 alunos da graduação que, enquanto atuaram no LUQS, defenderam os seus trabalhos de Conclusão de Curso, a maior parte deles, em Ciência da Computação. Também participaram 31 alunos de graduação e 4 alunos do ensino médio em pesquisas de iniciação científica, com bolsas financiadas pelo CNPq, FUNCAP, Fundação Edson Queiroz e Empresas. Destaca-se, também, mais 37 alunos de mestrado e 5 alunos de doutorado. Dentre esses alunos de pósgraduação stricto sensu, 38 foram alunos do PPGIA-UNIFOR, e somente 4 da Universidade Federal do Ceará - UFC.

O LUQS atua com 4 principais laboratórios parceiros: Laboratório de Pesquisa e Inovação - LAPIN (UNIFOR), na coordenação dos Professores Daniel Chagas e Daniel Valente; o Laboratório de Engenharia do Conhecimento - LEC (UNIFOR), na coordenação dos Professores de Inteligência Artificial: Vasco Furtado, Carlos Caminha Neto e Vládia Pinheiro; O Laboratório Interdisciplinar de Computação e Engenharia de Software - LINCE (UFC), na coordenação da Profa. Marília Mendes e o Laboratório MULTIMEIOS (UFC/FACED), na coordenação do prof. Hermínio Borges Neto. Há ainda a parceria com vários pesquisadores nacionais e internacionais. São grupos de pesquisa dinâmicos, que colaboram conforme a demanda. Os principais resultados das parcerias são encontrados descritos em artigos e relatórios de projetos.

As principais contribuições das ações e pesquisas foram: (1) Promoção do capítulo da ACM SIGCHI, situado em Fortaleza, chamado Br-CHI, de 2010-2016, realizando ações para aproximação entre Empresas e Academia e para divulgação dos estudos da área de IHC e das oportunidades proporcionadas pelas associações SBC e ACM; (2) Organização dos seguintes eventos: IHC 2002 em Fortaleza; CLIHC 2009 em Mérida, México; Competição de avaliação no IHC 2008 e; Workshop de Ensino de IHC utilizando Metodologias Ágeis no IHC 2018; (3) Realização do dia da Usabilidade (WUD) em 2008, 2009 e 2010 em parceria com o Instituto Atlântico; (4) Lançamento de um livro em 2012, contendo a descrição das principais pesquisas sobre avaliação de sistemas, realizadas no LUQS [8]; (5) Participação para as discussões sobre os Grandes Desafios para as pesquisas de IHC em 2014, com a escrita de artigos para 3 dos 5 temas apresentados no referido documento; bem como em 2017; (6) Contribuição social 
para a interiorização de pesquisadores de IHC em cidades do Estado do Ceará, ajudando na disseminação de conhecimentos na área. Desde a criação do grupo, três ex-alunos, atualmente, doutores, são professores em Russas (cidade a $167 \mathrm{Km}$ de Fortaleza) e em Jaguaribe $(300 \mathrm{Km}$ da Capital). Eles contribuem como coorientadores e parceiros do LUQS; (7) Aproximação das pesquisas de IHC com a prática realizada em empresas, por meio da promoção de eventos, na cidade de Fortaleza, Cuiabá e Belém. Foram ações realizadas em 2013 e 2014 com apoio financeiro do SIGCHI/ACM; e (8) Os pesquisadores parceiros do LUQS apoiam o projeto Mulheres na Ciência, lançado pela Unifor, participando das iniciativas: vídeos para motivação de mulheres se integrarem à pesquisa, lançados no dia internacional da mulher, em 2019 (https://youtu.be/pZ-E00vFFOk); e palestras em seminários, promovidos pelos cursos de psicologia e de computação.

\section{Pesquisadores / Desenvolvedores envolvidos}

Historicamente, o grupo que atua no LUQS teve a colaboração de diversos pesquisadores nacionais e internacionais desde sua criação. Atualmente, os seguintes professores realizam trabalhos juntos: 7 professores da UNIFOR (Elizabeth Sucupira Furtado (D.Sc), Plácido Rogério (Dr.), Vasco Furtado (D.Sc), Rafaela Ponte Lisboa (Dra.), Julio Guido Militão (Mestre), Daniel Chagas (Mestre) e Liádina Camargo (Mestre)), bem como 5 professores colaboradores de outras instituições (Marília Soares Mendes (Dra, UFC), Patrícia Vasconcelos (Dra, UFC), Virgílio Almeida (Dr, UFMG/Harward), Denilson Carneiro (Dr, IFCE) e Hermínio Borges Neto (Dr, UFC/FACED)). Para o desenvolvimento e execução dos projetos, atualmente tem-se no LUQS, 4 alunos de doutorado, 5 alunos de mestrado e 6 alunos de graduação. Porém, cada laboratório, parceiro do LUQS, conta com sua própria equipe.

\section{Projetos de Pesquisa}

Os principais projetos (concluídos/em andamento) e os seus respectivos financiadores são descritos como segue. A lista completa se encontra no lattes da profa. coordenadora do LUQS. São eles: (1) Projeto SBTVD (2005) -Finep; (2) Projeto SAMBA para TVD (2007-2009) - Financiamento Europeu; (3) Projeto para elaboração de um Programa de Audiência para TVD (2011) Finep; (4) Projeto para EaD via TVD (2009-2012)-Capes e Funcap; (5) Projeto mobilidade urbana (2012)-Funcap e Fotosensores; (6) Institucionalizando a usabilidade nas perspectivas da inovação dos processos de software (2010-2013)-CNPq; (7) Projeto para Trabalho Flexível na Companhia de Energia Elétrica do Ceará (2012-2014) - Anatel; (8) Disseminação do conhecimento em IHC (2013-2018) - CNPq; (9) Verbup news (2018) - Funcap e Empresa Verbup; (10) Análise das interfaces do sistema de Roteirização da Empresa PATHFIND (2018 - atual) - Financiamento PATHFIND; (11) Projeto de uso de interfaces tangíveis no ensino de pensamento computacional (2018 - atual) - Funcap e Fundação Edson Queiroz; (11) Análise de Viés da interação com assistentes inteligentes (2018-atual) - Fundação Edson Queiroz.

As linhas de pesquisa realizadas são: (1) Estudos sobre os usuários para e/ou em experiências com produtos interativos; (2) Métodos de avaliação das experiências dos usuários com produtos interativos; (3) Modelos de dados para análise e projeto da interação de sistemas; (4) Inovação de produtos e de processos de Software, e (5) Ensino de IHC.

As principais ferramentas destinadas para contribuir com temas e assuntos de interesse da área IHC desenvolvidas pelo grupo são: (1) UxMarks [3]: é um SS para a aprendizagem de conceitos e práticas de IHC/UX que agrega conteúdos relativo a IHC de grupos temáticos nas redes sociais como o Facebook e depois disponibiliza esse conteúdo livremente para a comunidade de interessados na área, de forma que eles possam classificá-lo no estilo Wiki; e (2) UUX-Posts $[16,18]$ : apoia a avaliação textual de Sistemas Sociais ou outros sistemas nos quais possam ser obtidos textos dos usuários. A ferramenta extrai e/ou analisa as opiniões dos usuários a respeito do sistema em uso. Ela está constantemente sendo atualizada a partir das pesquisas do grupo, relacionadas a IHC e IA.

\section{Infraestrutura disponível para a execução das pesquisas}

O LUQS faz parte dos espaços de inovação da Unifor [21] e se localiza integrado ao parque tecnológico da Unifor, que é destinado à instalação de empresas com seus setores de PDI (Pesquisa Desenvolvimento e Inovação), incubadoras de empresa, além de laboratórios de inovação a serem compartilhados pelas empresas. Está localizado majoritariamente no Bloco M. As empresas instaladas no espaço têm a oportunidade de, por meio do desenvolvimento de projetos conjuntos, beneficiar-se da capacidade científica e técnica dos pesquisadores da instituição para a criação de soluções inovadoras a problemas correntes, bem como para criação de inovações que conquistem novos mercados. O parque tem atualmente 14 empresas e também o comitê de ética, favorecendo ao LUQS, a realização de consultorias e a aprovação ética dos experimentos realizados, respectivamente.

O LUQS possui uma estrutura própria para a avaliação de sistemas interativos, mas conta também com a estrutura compartilhada no parque, para a realização de reuniões e desenvolvimento de software. A Figura 1 ilustra a sala onde alguns tipos de testes de usabilidade são realizados, com ou sem usuários, e a Figura 2 ilustra a sala utilizada para observação e análise dos resultados desses tipos de testes. Outros tipos de experimentos, nos quais aspectos dinâmicos e contextuais são investigados, também são realizados. Nesses casos, os experimentos com usuários são feitos em locais variados, inclusive em escolas.

\section{Soluções desenvolvidas}

As publicações mais citadas, segundo o Google Scholar dos pesquisadores são: (1) Geração de interfaces baseadas em modelos de interação [9,12, 13]; (2) Integração de práticas de IHC em processos de Engenharia de Software [14, 6]; (3) Geração do design da interação usando multi-critérios [17,5]; (4) Estudo da Emoção do usuário em experiências hedônicas via medidas psicofisiológicas [2]; e (5) Análise de texto para avaliar a qualidade das experiências dos usuários em sistemas interativos [18] e [7].

Dois principais prêmios obtidos no LUQS foram os seguintes: O TVILO system [11], prêmio de "melhores ferramentas" com um sistema para suportar a educação a distância no Workshop de 
Ferramentas e aplicações - Webmedia, em Belo Horizonte - MG; e o prêmio Prof. Arnaldo Dias Belchior, obtido no Concurso de “Teses e Dissertações em Qualidade de Software" [19, 20].

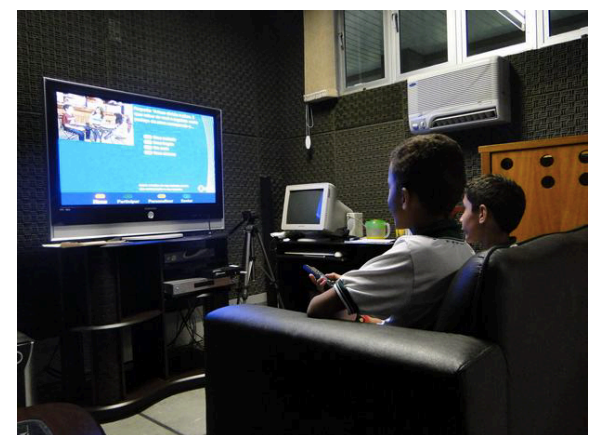

Figura 1. Sessão de teste de usabilidade para TV Digital

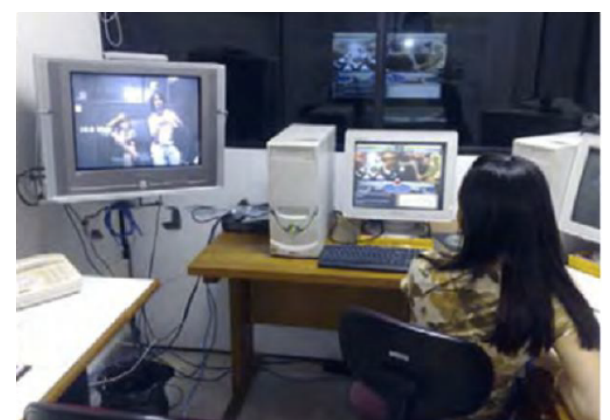

Figura 2. Teste de usabilidade - sala de observação.

\section{Interesses de parcerias com outros grupos}

Os principais interesses são agrupados em dois itens e estão descritos nesta seção, juntamente com os desafios, que motivam a necessidade de parcerias.

Interesse 1: Devido à localização do laboratório apresentado e de seus parceiros serem localizados na região Nordeste, os experimentos são pouco diversificados para representar o Brasil. Interessa-nos parcerias com pesquisadores localizados em outras regiões Brasileiras, para estudar usuários com variações sócioculturais-econômicas. Idealizamos as parcerias para a realização de experimentos para os devidos fins: (1) Avaliar a interação via voz com perfis diversos de usuários ao usarem assistentes inteligentes [15]. Aspectos linguísticos e de sotaque, associados à outros fatores como escolaridade, idade e gênero deverão ser investigados em outros tipos de usuários; e (2) Avaliar a aprendizagem do pensamento computacional de usuários crianças com o uso da interface de programação tangível Code Domino [4]. Estudos sobre replicabilidade de soluções de ensino de pensamento computacional, uso de soluções em diferentes faixas etárias (alfabetização, crianças, adolescentes e em nível universitário) e impacto de soluções de interação em questões de gênero no ensino STEM são áreas de interesse para expansão da pesquisa.

Interesse 2: Sendo IHC uma área multidisciplinar, interessa-nos obter parcerias com pesquisadores da área: a) de Sistemas Colaborativos (SiCos), que trabalhe com redes de relacionamento, compartilhamento de conteúdo e colaboração; b) de Processamento da Linguagem Natural (PLN) que tenham interesse em apoiar a interpretação da linguagem dos usuários em sistemas, envolvendo técnicas de pré-processamento, como limpeza de textos e análises morfológicas, sintáticas e semânticas; e c) de IA, especificamente Mineração de dados e aprendizagem de máquina que se interessem por análise de sentimentos para identificação das postagens negativas, positivas e neutras encontradas em uma avaliação textual. Idealizamos as parcerias para os devidos fins: (1) Extensão ou uso de modelos de interação: MALTU [18] e MASSVA [7] em outros tipos de sistemas interativos; (2) Estudos sobre usuários do Sistema por meio de seus textos; (3) Estudo e aplicação de técnicas de PLN, Mineração de Dados e Aprendizado de Máquina para avaliação de sistemas $[1,10]$;

\section{REFERÊNCIAS}

[1] Afonso M. S. Lima, Marilia S. Mendes, and Lívia A. Cruz. 2019. Enrichment of dictionaries to improve the automatic classification of feelings in postings related to the use of systems. In SBSI 2019. ACM, 10.

[2] Camila Maia, Elizabeth S. Furtado., A framework for user's emotional evaluation based on psychophysiological measures. In IHC'2017;

[3] Carlos R. Carvalho, Elizabeth S. Furtado. Wikimarks: an approach proposition for generating collaborative, structured content from social networking sharing on the web. (IHC 2012), Cuiabá, MT, Brasil, p. 95-98, novembro, 2012.

[4] Daniel A. Chagas, Elizabeth S. Furtado; Camila M. Moreira; Plácido R. Pinheiro. Analyzing the Multicriteria of the Interaction Design of an Educational Map Application for Digital TV from User Preferences. 2015. Journal of Universal Computer Science (Print), v. 21, p. 1470-1481, 2015;

[5] Daniel A. Chagas, Rafaela P. Lisboa, Elizabeth S. Furtado. 2008. Interfaces adaptativas tangíveis e virtuais para ensino de lógica. IN WTD in IHC'2018;

[6] David Barbosa, Elizabeth S. Furtado, Albert Schilling. 2008. Uma Estratégia de Apoio à Institucionalização da Usabilidade em Ambientes de Desenvolvimento Ágil. In IHC'2008;

[7] DC OLIVEIRA. MASSVA: Modelo de Avaliação de Sistemas Sociais sob a ótica dos Valores humanos a partir das postagens dos usuários. Tese (doutorado) Unifor. PPGIA, 2018;

[8] Elizabeth S. Furtado (Org.). 2012. Qualidade da interação de sistemas e novas abordagens para a avaliação. Curitiba: CRV, Brasil.

[9] Elizabeth S. Furtado, Vasco Furtado, silva w., rodrigues,d., taddeo, 1., Limbourg Q., Vanderdonckt, J. An ontology-based method for universal design of user interfaces. 2001.

[10] Hismael C. Oliveira, Alexandre M. Arruda, Marília S. Mendes. 2019. Automatic Identification of Postings Related to the Use Through Deep Learning Models. (BRACIS - ENIAC 2019).

[11] Italo Matos; Elizabeth S. Furtado. TvILOTool - Uma Ferramenta Orientada Serviços para Produção de Conteúdos para Televisão Digital Brasileira baseada em Objetos de Aprendizagem. In: Webmedia. BH. 2010. Disponível em: http://luqs.unifor.br/wp-content/uploads/2010/12/webmidia-v16.pdf;

[12] Júlio G. Militao, Elizabeth S. Furtado, Ítalo Matos. Association between Requirements of Interaction and Educational Contents of iDTV applications and UsiXML models by addressing the need to structure TVilos in Crossmedia. In: USIXML, 2010, Alemanha..v. 1. p. 83-89;

[13] Junia Gonçalves, Elizabeth S. Furtado. 2013. Um framework para aprimoramento do processo de inovação: estudo de caso em design universal. In IHC 2013.

[14] Kênia Sousa e Elizabeth S. Furtado. 2003. RUPi-A Unified Process that Integrates Human-Computer Interaction and Software Engineering. ICSE Workshop on SEHCI.

[15] Lanna Sales, Elizabeth S. Furtado, Vasco Furtado, Virgílio Almeida. 2019. Investigações sobre Discriminação em Assistentes de Voz Inteligentes. In IHC'2019 (in press);

[16] Marília S. Mendes and Elizabeth S. Furtado. 2017. UUX-Posts: A Tool for Extracting and Classifying Postings Related to the Use of a System. In CLIHC17.

[17] Marília S. Mendes, Ana L. Carvalho, Elizabeth S. Furtado, Plácido Pinheiro. 2009. A co-evolutionary interaction design of digital TV applications based on verbal decision analysis of user experiences. In International Journal of Digital Culture and Electronic Tourism.

[18] MS Mendes. 2015. MALTU-Model for evaluation of interaction in social systems from the Users Textual Language. 200 f. Ph.D. Dissertation. thesis (Ph. D in computer science)-Federal University of Ceará (UFC)

[19] Sofia Marçal, freitas, b., soares f, elizabeth sucupira furtado, maciel, t., belchior a., Blending Scrum practices and CMMI project management process areas in Journal of Innovations in Systems and Software Engineering. Vol 4., 17-29. 2008 ;

[20] Sofia Marçal; Elizabeth S. Furtado. Scrummi: Um processo de gestão ágil baseado no Scrum e aderente ao CMMI, concurso de tese SBQS'2010;

[21] Unifor - Espaços de Inovação. Disponível em: $<$ https://www.unifor.br/web/pesquisa-inovacao/espacos-de-inovacao $>$. Acesso em 7 set 2019. 